\title{
PATIENT SAFETY IN INTENSIVE CARE UNIT SILOAM HOSPITALS MANADO, INDONESIA: AN OVERVIEW
}

\author{
Adejohn Nursalim, Fransien Virginia Tamusa, Lady Galatia Lapian \\ Hospital Administration, Department of Public Health Science, Sam Ratulangi University \\ Email: ladygalatia@yahoo.co.id
}

\begin{abstract}
Background: The intensive care unit (ICU) is a complex and dynamic environment. Mistake does occur, sometimes with severe consequences. The most common errors reported are concerning airway management and invasive lines, tubes and drains.

Methods: This research comprised of quantitative review methods to overview the implementation of patient safety and ICU. This research used descriptive research design while investigation underwent within the ward for one week. Data extraction process used questionnaire method to involve all staff members within ICU of Siloam Hospitals Manado.

Results: Cronbach's alpha shows result of 0.854, which is higher than $r$ table for $n$ 96. The questionnaire was deemed reliable. Research showed that ICU staffs have good knowledge, attitude, competencies, situation awareness, leadership, communication, teamwork and team leadership as well as decision making related to patient safety. Moreover, ICU staff was burntout due to high workload, more than each staff can bear. Further result showed that fire prevention, life saving control, room and equipments, chemicals and dangerous object safety, management of medical equipments, electrical handling, patient safety, storage cupboard usage and medication management all have good policy and procedure. On the other hand, infection control procedure needs to be improved.

Conclusion: From this research, it is concluded that ICU Siloam Hospitals Manado has applied patient safety principles' well. For further improvements, the management should consider to conduct trainings to staffs regarding patient safety.
\end{abstract}

Keywords: patient safety, ICU

\section{Introduction}

Many research in healthcare have shown patients to frequently experience unnecessary harm as a result of preventable medical errors. ${ }^{1}$ These errors can result in unnescessary suffering to the patients including high financial cost caused by extended hospital stays.

The intensive care unit (ICU) has a complex and dynamic environment. ${ }^{2}$ Typically, patients in the ICU have been shown to be particularly susceptible to experiencing an error. An error made in intensive care unit is defined as any incident whichcould have affectedthe safety of the patient while under ICU management. ${ }^{3} \mathrm{~A}$ recent review concluded that to reduce the incidence of the events will require the identification of the causes and development of methods that will prevent errors or reduce their effect. ${ }^{4}$

Siloam Hospital is a private hospital with 10 ICU beds. In average, there are 10 (ten) nurses working in the unit each shift, or approximately 3:1 patient-nurse ratio. Most nurses in Siloam Hospital have high capability in theory and skill informal educational degree qualification (diploma to bachelor degree), in addition to specialized qualifications, such as Basic Cardiology Training, Service Improvement, Fire Safety and Emergency Drill, Maternity Training, Phlebotonomy Training, Basic Intensive Care Unit Nurses Training and Comprehensive Emergency Obstetric and Neonatal Training and ACLS. Numbers of patient ICU admission in 2014 is 265 patients. Numbers of 
patient death in ICU in 2014 is 49 deaths. There are two incidents of medication error in 2014 regarding late medication administration for more than 2 hours. The cause was identified for both incidents, which were due to empty stock in the pharmacy. No reports related to post-operative infection, ventilator associated pneumonia and falls, but one case of pressure injury was reported in hospital documentation.

The aims of the study were to overview the implementation of patient safety in intensive care unit and to develop strategies in order to prevent reoccurence of errors, thus to decrease possible morbidity and mortality.

\section{Research Method}

This study conducted at a private hospital: Siloam Hospitals Manado. This study was approved by Postgraduate study of Public Health Universitas Sam Ratulangi Manado and Siloam Hospitals Manado.The intensive care unit in this hospital is a closed unit with multidisciplinary team approach in providing patient care. Respondents used in this research are the members of the multidisciplinary itself andimplied consent attained from them upon returning the survey.

This research uses descriptive research design during investigation within the ward for one week (March $23^{\text {rd }}$ to March $30^{\text {th }}$ 2015). Data extraction process used questionnaire method to involve all staff members within ICU of Siloam Hospital Manado. The questionnaire discusses about some identified factors associated to patient safety; teamwork climate, job satisfaction, perception of hospital management, safety climate, working condition and stress recognition. ${ }^{5}$ Response is given through 5 likert scale, in which 1 is strongly disagree to 5 is strongly agree, Gutmann Scale and the language used is Indonesian.

\section{Results and Analysis}

Ayuwardhani S stated factors associated to patient safety; teamwork climate, job satisfaction, perception of hospital management, safety climate, working condition and stress recognition related to patient safety in hospital. Ideally, each factor related to patient safety are met with good. ${ }^{5}$

Implementation of factors related to patient safety in hospitals can be judged by hospital staff and also outsiders. With this research method, assessment of fators related to patient safety obtained from staff in hospital especially in intensive care unit.

According to Table 1, can be told that all questions in the questionnaire are valid. Questions regarding staff knowledge of patient safety number 3, 4, 6, 7 and 11 are valid. Majority ofquestions in staff attitude category are valid, except question number 7 . Further, valid questions regarding staff motivation are questions number 6 , $9,10,11$ and 12 . 
Table 1. Validity

\begin{tabular}{|c|c|c|c|c|c|c|c|c|c|}
\hline & & Knowledge & Attitude & Motivation & Competencies & Awareness & Stress & Fatigue & Leadership \\
\hline \multirow[t]{3}{*}{1} & $\begin{array}{l}\text { Pearson } \\
\text { Correlation }\end{array}$ & & 0.747 & 0.270 & 0.683 & 0.762 & 0.378 & 0.680 & 0.770 \\
\hline & Sig. & & & 0.156 & 0.002 & 0.000 & 0.075 & 0.002 & 0.000 \\
\hline & Pearson & $\begin{array}{c}16 \\
0.356\end{array}$ & $\begin{array}{c}16 \\
0.805\end{array}$ & $\begin{array}{c}16 \\
0.361\end{array}$ & $\begin{array}{c}16 \\
0.651\end{array}$ & $\begin{array}{c}16 \\
0.617\end{array}$ & 0.232 & 0.137 & 0.828 \\
\hline 2 & $\begin{array}{l}\text { Correlation } \\
\text { Sig. } \\
N\end{array}$ & $\begin{array}{c}0.088 \\
16\end{array}$ & 16 & $\begin{array}{c}0.085 \\
16\end{array}$ & $\begin{array}{c}0.03 \\
16\end{array}$ & $\begin{array}{c}0.005 \\
16\end{array}$ & $\begin{array}{c}0.193 \\
16\end{array}$ & $\begin{array}{c}0.306 \\
16\end{array}$ & $\begin{array}{c}0.000 \\
16\end{array}$ \\
\hline \multirow[t]{2}{*}{3} & $\begin{array}{l}\text { Pearson } \\
\text { Correlation }\end{array}$ & 0.487 & 0.581 & 0.350 & 0.845 & 0.265 & 0.425 & 0.402 & 0.685 \\
\hline & $\begin{array}{l}\text { Sig. } \\
N\end{array}$ & $\begin{array}{c}0.028 \\
16\end{array}$ & $\begin{array}{c}0.009 \\
16\end{array}$ & $\begin{array}{c}0.092 \\
16\end{array}$ & $\begin{array}{c}0.000 \\
16\end{array}$ & $\begin{array}{c}0.160 \\
16\end{array}$ & $\begin{array}{c}0.050 \\
16\end{array}$ & $\begin{array}{c}0.061 \\
16\end{array}$ & $\begin{array}{c}0.002 \\
16\end{array}$ \\
\hline \multirow[t]{2}{*}{4} & $\begin{array}{l}\text { Pearson } \\
\text { Correlation }\end{array}$ & 0.755 & 0.517 & 0.137 & 0.798 & 0.707 & 0.249 & 0.713 & 0.630 \\
\hline & $\begin{array}{l}\text { Sig. } \\
\text { N }\end{array}$ & 16 & $\begin{array}{c}0.020 \\
16\end{array}$ & $\begin{array}{c}0.307 \\
16\end{array}$ & $\begin{array}{c}0.000 \\
16\end{array}$ & $\begin{array}{c}0.001 \\
16\end{array}$ & $\begin{array}{c}0.176 \\
16\end{array}$ & $\begin{array}{c}0.001 \\
16\end{array}$ & $\begin{array}{c}0.004 \\
16\end{array}$ \\
\hline \multirow[t]{2}{*}{5} & $\begin{array}{l}\text { Pearson } \\
\text { Correlation }\end{array}$ & & 0.803 & 0.216 & 0.428 & & & & 0.345 \\
\hline & $\begin{array}{l}\text { Sig. } \\
N\end{array}$ & 16 & 16 & $\begin{array}{c}0.211 \\
16\end{array}$ & $\begin{array}{c}0.049 \\
16\end{array}$ & & & & $\begin{array}{c}0.095 \\
16\end{array}$ \\
\hline \multirow[t]{2}{*}{6} & $\begin{array}{l}\text { Pearson } \\
\text { Correlation }\end{array}$ & 0.535 & 0.590 & 0.474 & & & & & \\
\hline & $\begin{array}{l}\text { Sig. } \\
\text { N }\end{array}$ & $\begin{array}{c}0.016 \\
16\end{array}$ & $\begin{array}{c}0.008 \\
16\end{array}$ & $\begin{array}{c}0.032 \\
16\end{array}$ & & & & & \\
\hline \multirow[t]{2}{*}{7} & $\begin{array}{l}\text { Pearson } \\
\text { Correlation }\end{array}$ & 0.535 & 0.029 & 0.279 & & & & & \\
\hline & $\begin{array}{l}\text { Sig. } \\
\text { N }\end{array}$ & $\begin{array}{c}0.016 \\
16\end{array}$ & $\begin{array}{c}0.457 \\
16\end{array}$ & $\begin{array}{c}0.147 \\
16\end{array}$ & & & & & \\
\hline \multirow[t]{2}{*}{8} & $\begin{array}{l}\text { Pearson } \\
\text { Correlation }\end{array}$ & & 0.321 & 0.020 & & & & & \\
\hline & $\begin{array}{l}\text { Sig. } \\
\text { N }\end{array}$ & 16 & $\begin{array}{c}0.113 \\
16\end{array}$ & $\begin{array}{l}0.471 \\
16\end{array}$ & & & & & \\
\hline \multirow[t]{2}{*}{9} & $\begin{array}{l}\text { Pearson } \\
\text { Correlation }\end{array}$ & & & 0.527 & & & & & \\
\hline & $\begin{array}{l}\text { Sig. } \\
N\end{array}$ & 16 & & $\begin{array}{c}0.018 \\
16\end{array}$ & & & & & \\
\hline \multirow[t]{2}{*}{10} & $\begin{array}{l}\text { Pearson } \\
\text { Correlation }\end{array}$ & 0.243 & & 0.552 & & & & & \\
\hline & $\begin{array}{l}\text { Sig. } \\
N\end{array}$ & $\begin{array}{c}0.182 \\
16\end{array}$ & & $\begin{array}{c}0.013 \\
16\end{array}$ & & & & & \\
\hline \multirow[t]{2}{*}{11} & $\begin{array}{l}\text { Pearson } \\
\text { Correlation }\end{array}$ & 0.487 & & 0.429 & & & & & \\
\hline & $\begin{array}{l}\text { Sig. } \\
\mathrm{N}\end{array}$ & $\begin{array}{c}0.028 \\
16\end{array}$ & & $\begin{array}{c}0.048 \\
16\end{array}$ & & & & & \\
\hline \multirow[t]{3}{*}{12} & $\begin{array}{l}\text { Pearson } \\
\text { Correlation }\end{array}$ & & & 0.802 & & & & & \\
\hline & Sig. & & & 0.000 & & & & & \\
\hline & $\mathrm{N}$ & 16 & & 16 & & & & & \\
\hline
\end{tabular}

While all of questions regarding staff competency are valid, no legible questions under staff stress level. Question number 1, 2, and 4 are effective to represent staff's condition alertness. Under staff burnout level only has 2 valid question, they are question number 1 and 4 . The last category in table 1, which is leadership has 4 solid question which are number 1 to 4 . 
Table 2. Questionnaire Validity

\begin{tabular}{|c|c|c|c|c|c|}
\hline & & Communication & $\begin{array}{l}\text { Team } \\
\text { Work }\end{array}$ & $\begin{array}{c}\text { Team } \\
\text { Leadership }\end{array}$ & $\begin{array}{c}\text { Decision } \\
\text { Making }\end{array}$ \\
\hline \multirow[t]{2}{*}{1} & $\begin{array}{l}\text { Pearson } \\
\text { Correlation }\end{array}$ & 0.644 & 0.069 & 0.677 & 0.338 \\
\hline & $\begin{array}{l}\text { Sig. } \\
\text { N }\end{array}$ & $\begin{array}{c}0.004 \\
16\end{array}$ & $\begin{array}{c}0.399 \\
16\end{array}$ & $\begin{array}{c}0.002 \\
16\end{array}$ & $\begin{array}{c}0.100 \\
16\end{array}$ \\
\hline \multirow[t]{2}{*}{2} & $\begin{array}{l}\text { Pearson } \\
\text { Correlation }\end{array}$ & 0.396 & 0.857 & 0.570 & 0.244 \\
\hline & $\begin{array}{l}\text { Sig. } \\
\text { N }\end{array}$ & $\begin{array}{c}0.065 \\
16\end{array}$ & $\begin{array}{c}0.000 \\
16\end{array}$ & $\begin{array}{c}0.011 \\
16\end{array}$ & $\begin{array}{c}0.181 \\
16\end{array}$ \\
\hline \multirow[t]{2}{*}{3} & $\begin{array}{l}\text { Pearson } \\
\text { Correlation }\end{array}$ & 0.366 & 0.587 & 0.754 & 0.840 \\
\hline & $\begin{array}{l}\text { Sig. } \\
\mathrm{N}\end{array}$ & $\begin{array}{c}0.065 \\
16\end{array}$ & $\begin{array}{c}0.008 \\
16\end{array}$ & $\begin{array}{c}0.000 \\
16\end{array}$ & $\begin{array}{c}0.000 \\
16\end{array}$ \\
\hline \multirow[t]{2}{*}{4} & $\begin{array}{l}\text { Pearson } \\
\text { Correlation }\end{array}$ & 0.396 & 0.491 & 0.800 & 0.683 \\
\hline & $\begin{array}{l}\text { Sig. } \\
\mathrm{N}\end{array}$ & $\begin{array}{c}0.064 \\
16\end{array}$ & $\begin{array}{c}0.027 \\
16\end{array}$ & $\begin{array}{c}0.000 \\
16\end{array}$ & $\begin{array}{c}0.002 \\
16\end{array}$ \\
\hline \multirow[t]{2}{*}{5} & $\begin{array}{l}\text { Pearson } \\
\text { Correlation }\end{array}$ & 0.280 & 0.652 & 0.528 & \\
\hline & $\begin{array}{l}\text { Sig. } \\
\mathrm{N}\end{array}$ & $\begin{array}{c}0.146 \\
16\end{array}$ & $\begin{array}{c}0.003 \\
16\end{array}$ & $\begin{array}{c}0.018 \\
16\end{array}$ & \\
\hline \multirow[t]{2}{*}{6} & $\begin{array}{l}\text { Pearson } \\
\text { Correlation }\end{array}$ & 0.847 & & 0.525 & \\
\hline & $\begin{array}{l}\text { Sig. } \\
\text { N }\end{array}$ & $\begin{array}{c}0.000 \\
16\end{array}$ & & $\begin{array}{c}0.018 \\
16\end{array}$ & \\
\hline \multirow[t]{2}{*}{7} & $\begin{array}{l}\text { Pearson } \\
\text { Correlation }\end{array}$ & 0.867 & & & \\
\hline & $\begin{array}{l}\text { Sig. } \\
\mathrm{N}\end{array}$ & $\begin{array}{c}0.000 \\
16\end{array}$ & & & \\
\hline 8 & $\begin{array}{l}\text { Pearson } \\
\text { Correlation } \\
\text { Sig. } \\
\mathrm{N}\end{array}$ & $\begin{array}{c}0.846 \\
0.000 \\
16 \\
\end{array}$ & & & \\
\hline
\end{tabular}

Table 2 further describes questions that used in the questionnaire. Questions number 1, 6, 7 and 8 are valid to enquire regarding staff communication in relation to patient safety. Staff teamwork question from number 2 to 5.While all of questions regarding team leadership are valid, only question number 3 and 4 are valid under decision making category.

Table 3. Validity of Hospital Environment Category

\begin{tabular}{|c|c|c|c|c|c|c|c|}
\hline & & $\begin{array}{c}\text { Fire } \\
\text { Prevention }\end{array}$ & $\begin{array}{c}\text { Infection } \\
\text { Control } \\
\end{array}$ & $\begin{array}{l}\text { Life Saving } \\
\text { Control }\end{array}$ & $\begin{array}{l}\text { Room and } \\
\text { Equipment }\end{array}$ & $\begin{array}{l}\text { Dangerous } \\
\text { Substance }\end{array}$ & $\begin{array}{c}\text { Medical } \\
\text { Equipment }\end{array}$ \\
\hline \multirow[t]{2}{*}{1} & $\begin{array}{l}\text { Pearson } \\
\text { Correlation }\end{array}$ & 0.820 & 0.494 & 0.700 & 0.931 & 0.845 & 0.770 \\
\hline & $\begin{array}{l}\text { Sig. } \\
N\end{array}$ & $\begin{array}{c}0.000 \\
16\end{array}$ & $\begin{array}{c}0.026 \\
16\end{array}$ & $\begin{array}{c}0.001 \\
16\end{array}$ & $\begin{array}{c}0.000 \\
16\end{array}$ & $\begin{array}{c}0.000 \\
16\end{array}$ & $\begin{array}{c}0.000 \\
16\end{array}$ \\
\hline \multirow[t]{2}{*}{2} & $\begin{array}{l}\text { Pearson } \\
\text { Correlation }\end{array}$ & 0.584 & 0.712 & 0.816 & 0.808 & 0.346 & 0.770 \\
\hline & $\begin{array}{l}\text { Sig. } \\
\text { N }\end{array}$ & $\begin{array}{c}0.000 \\
16\end{array}$ & $\begin{array}{c}0.001 \\
16\end{array}$ & $\begin{array}{c}0.000 \\
16\end{array}$ & $\begin{array}{c}0.000 \\
16\end{array}$ & $\begin{array}{c}0.094 \\
16\end{array}$ & $\begin{array}{c}0.000 \\
16\end{array}$ \\
\hline \multirow[t]{2}{*}{3} & $\begin{array}{l}\text { Pearson } \\
\text { Correlation }\end{array}$ & 0.821 & 0.197 & 0.883 & & & 0.305 \\
\hline & $\begin{array}{l}\text { Sig. } \\
N\end{array}$ & $\begin{array}{c}0.000 \\
16\end{array}$ & $\begin{array}{c}0.233 \\
16\end{array}$ & 16 & & 16 & $\begin{array}{c}0.126 \\
16\end{array}$ \\
\hline \multirow[t]{2}{*}{4} & $\begin{array}{l}\text { Pearson } \\
\text { Correlation }\end{array}$ & 0.736 & & & & 0.808 & \\
\hline & $\begin{array}{l}\text { Sig. } \\
\mathrm{N}\end{array}$ & $\begin{array}{c}0.001 \\
16\end{array}$ & 16 & & & $\begin{array}{c}0.000 \\
16\end{array}$ & \\
\hline
\end{tabular}

KESMAS Vol. 9, No. 2, September 2015 : 101 - 108 


\begin{tabular}{|c|c|c|c|c|c|c|c|}
\hline & & $\begin{array}{c}\text { Fire } \\
\text { Prevention }\end{array}$ & $\begin{array}{c}\text { Infection } \\
\text { Control }\end{array}$ & $\begin{array}{l}\text { Life Saving } \\
\text { Control }\end{array}$ & $\begin{array}{l}\text { Room and } \\
\text { Equipment }\end{array}$ & $\begin{array}{l}\text { Dangerous } \\
\text { Substance }\end{array}$ & $\begin{array}{c}\text { Medical } \\
\text { Equipment }\end{array}$ \\
\hline \multirow[t]{2}{*}{5} & Pearson & 0.655 & 0.176 & & & & \\
\hline & Sig. & 0.003 & 0.257 & & & & \\
\hline \multirow[t]{2}{*}{6} & $\begin{array}{l}\text { Pearson } \\
\text { Correlation }\end{array}$ & 0.459 & 0.321 & & & & \\
\hline & $\begin{array}{l}\text { Sig. } \\
N\end{array}$ & $\begin{array}{c}0.037 \\
16\end{array}$ & $\begin{array}{c}0.113 \\
16\end{array}$ & & & & \\
\hline \multirow[t]{2}{*}{7} & $\begin{array}{l}\text { Pearson } \\
\text { Correlation }\end{array}$ & 0.726 & 0.176 & & & & \\
\hline & $\begin{array}{l}\text { Sig. } \\
\text { N }\end{array}$ & $\begin{array}{c}0.001 \\
16\end{array}$ & $\begin{array}{l}0.257 \\
16\end{array}$ & & & & \\
\hline \multirow[t]{2}{*}{8} & $\begin{array}{l}\text { Pearson } \\
\text { Correlation }\end{array}$ & & 0.257 & & & & \\
\hline & $\begin{array}{l}\text { Sig. } \\
N\end{array}$ & & $\begin{array}{c}0.168 \\
16\end{array}$ & & & & \\
\hline \multirow[t]{2}{*}{9} & $\begin{array}{l}\text { Pearson } \\
\text { Correlation }\end{array}$ & & 0.500 & & & & \\
\hline & $\begin{array}{l}\text { Sig. } \\
\mathrm{N}\end{array}$ & & $\begin{array}{c}0.024 \\
16\end{array}$ & & & & \\
\hline 10 & $\begin{array}{l}\text { Pearson } \\
\text { Correlation } \\
\text { Sig. }\end{array}$ & & 16 & & & & \\
\hline 11 & $\begin{array}{l}\text { Pearson } \\
\text { Correlation } \\
\text { Sig. } \\
\text { N }\end{array}$ & & 16 & & & & \\
\hline \multirow[t]{2}{*}{12} & $\begin{array}{l}\text { Pearson } \\
\text { Correlation }\end{array}$ & & 0.693 & & & & \\
\hline & $\begin{array}{l}\text { Sig. } \\
N\end{array}$ & & $\begin{array}{c}0.001 \\
16\end{array}$ & & & & \\
\hline \multirow[t]{2}{*}{13} & $\begin{array}{l}\text { Pearson } \\
\text { Correlation }\end{array}$ & & 0.546 & & & & \\
\hline & & & $\begin{array}{c}0.014 \\
16\end{array}$ & & & & \\
\hline \multirow[t]{2}{*}{14} & $\begin{array}{l}\text { Pearson } \\
\text { Correlation }\end{array}$ & & 0.439 & & & & \\
\hline & $\begin{array}{l}\text { Sig. } \\
N\end{array}$ & & $\begin{array}{c}0.044 \\
16\end{array}$ & & & & \\
\hline
\end{tabular}

Table 4. Validity of Hospital Environment Category

\begin{tabular}{|c|c|c|c|c|c|c|}
\hline & & $\begin{array}{l}\text { Electricity } \\
\text { Handling }\end{array}$ & $\begin{array}{c}\text { Infection } \\
\text { Control }\end{array}$ & $\begin{array}{l}\text { Patient Safety } \\
\text { Regulation }\end{array}$ & $\begin{array}{c}\text { Cupboard } \\
\text { Usage }\end{array}$ & $\begin{array}{c}\text { Medicine } \\
\text { Mangement }\end{array}$ \\
\hline \multirow[t]{2}{*}{1} & $\begin{array}{l}\text { Pearson } \\
\text { Correlation }\end{array}$ & 0.733 & 0.494 & 0.474 & 0.655 & \\
\hline & $\begin{array}{l}\text { Sig. } \\
\text { N }\end{array}$ & $\begin{array}{c}0.001 \\
16\end{array}$ & $\begin{array}{c}0.026 \\
16\end{array}$ & $\begin{array}{c}0.032 \\
16\end{array}$ & $\begin{array}{c}0.003 \\
16\end{array}$ & 16 \\
\hline \multirow[t]{2}{*}{2} & $\begin{array}{l}\text { Pearson } \\
\text { Correlation }\end{array}$ & 0.748 & 0.712 & 0.269 & & \\
\hline & $\begin{array}{l}\text { Sig. } \\
\text { N }\end{array}$ & $\begin{array}{c}0.000 \\
16\end{array}$ & $\begin{array}{c}0.001 \\
16\end{array}$ & $\begin{array}{c}0.157 \\
16\end{array}$ & 16 & 16 \\
\hline \multirow[t]{2}{*}{3} & $\begin{array}{l}\text { Pearson } \\
\text { Correlation }\end{array}$ & & 0.197 & 0.610 & 0.655 & 1.000 \\
\hline & & & $\begin{array}{c}0.233 \\
16\end{array}$ & $\begin{array}{c}0.006 \\
16\end{array}$ & $\begin{array}{c}0.003 \\
16\end{array}$ & $\begin{array}{c}0.000 \\
16\end{array}$ \\
\hline \multirow[t]{2}{*}{4} & $\begin{array}{l}\text { Pearson } \\
\text { Correlation } \\
\text { Sig. }\end{array}$ & & & & & \\
\hline & $\mathrm{N}$ & & 16 & 16 & & 16 \\
\hline
\end{tabular}




\begin{tabular}{|c|c|c|c|c|c|c|}
\hline & & $\begin{array}{l}\text { Electricity } \\
\text { Handling }\end{array}$ & $\begin{array}{c}\text { Infection } \\
\text { Control }\end{array}$ & $\begin{array}{c}\text { Patient Safety } \\
\text { Regulation }\end{array}$ & $\begin{array}{l}\text { Cupboard } \\
\text { Usage }\end{array}$ & $\begin{array}{c}\text { Medicine } \\
\text { Mangement }\end{array}$ \\
\hline \multirow[t]{2}{*}{5} & $\begin{array}{l}\text { Pearson } \\
\text { Correlation }\end{array}$ & & 0.176 & 0.025 & & \\
\hline & $\begin{array}{l}\text { Sig. } \\
\mathrm{N}\end{array}$ & & $\begin{array}{c}0.257 \\
16\end{array}$ & $\begin{array}{c}0.464 \\
16\end{array}$ & & 16 \\
\hline \multirow[t]{2}{*}{6} & $\begin{array}{l}\text { Pearson } \\
\text { Correlation }\end{array}$ & & 0.321 & 0.323 & & \\
\hline & $\begin{array}{l}\text { Sig. } \\
\mathrm{N}\end{array}$ & & $\begin{array}{c}0.113 \\
16\end{array}$ & $\begin{array}{c}0.111 \\
16\end{array}$ & & 16 \\
\hline \multirow[t]{2}{*}{7} & $\begin{array}{l}\text { Pearson } \\
\text { Correlation }\end{array}$ & & 0.176 & & & \\
\hline & Sig. & & $\begin{array}{l}0.257 \\
16\end{array}$ & & & 16 \\
\hline \multirow[t]{3}{*}{8} & $\begin{array}{l}\text { Pearson } \\
\text { Correlation }\end{array}$ & & 0.257 & & & \\
\hline & Sig. & & 0.168 & & & \\
\hline & $\mathrm{N}$ & & 16 & & & 16 \\
\hline
\end{tabular}

Table 3 and 4 further describes questions that used in the questionnaire.Questions number 1 to 7 are valid to enquire regarding to fire prevention.Questions regarding infection control number 1, 2, 9, 12, 13, and 14 are valid. Questions number 1 to 3 regarding live safing control are valid. Questions regarding room and equipment 1 and 2 are valid. Valid questions in dangerous substance category are questions number 1 to 4 . Question number 1 and 2 are effective to represent medical equipment management.Questions number 1 and 2 are valid to enquire regarding electrical handling. Valid questions in patient safety policy category are questions number 1 and 3 . Questions regarding cupboard usage number 1 and 3 are valid. Further, the only valid question regarding medicine management is question number 3 .

Table 5. Reliability Statistics

\begin{tabular}{cc}
\hline Cronbach's Alpha & N of Items \\
\hline .854 & 96 \\
\hline
\end{tabular}

Cronbach's alpha shows result of 0.854 , which is higher than $r$ table for $n 96$. Therefore this questionnaire is deemed to be reliable.

Table 6. Variables Analysis Summary

\begin{tabular}{lccccccc}
\hline & Knowledge & Attitude & Motivation & Competencies & $\begin{array}{c}\text { Situation } \\
\text { awareness }\end{array}$ & Fatigue & Leadership \\
\hline Results & 1.375 & 1.395 & 3.85 & 1.875 & 2.395 & 2.125 & 1.875 \\
Expectation & 1.5 & 3 & 3 & 3 & 3 & 3 & 3 \\
Analysis & Good & Good & Not good & Good & Good & Good & Good \\
\hline
\end{tabular}

\begin{tabular}{lcccc}
\hline & Communication & Teamwork & $\begin{array}{c}\text { Teamwork } \\
\text { Leadership }\end{array}$ & $\begin{array}{c}\text { Decision } \\
\text { Making }\end{array}$ \\
\hline Results & 2.953 & 2.75 & 2.145 & 2.468 \\
Expectation & 3 & 3 & 3 & 3 \\
Analysis & Good & Good & Good & Good \\
\hline
\end{tabular}

Variables that are good are the staff knowledge, attitude, situation awareness, competency, fatigue, leadership, communication, teamwork, team leadership and decision making. Motivation competency seems to be worse than expected. 
Table 7. Hospital Environment Variables Analysis Summary

\begin{tabular}{|c|c|c|c|c|c|c|c|}
\hline & $\begin{array}{c}\text { Fire } \\
\text { Prevention }\end{array}$ & $\begin{array}{c}\text { Infection } \\
\text { Control } \\
\end{array}$ & $\begin{array}{l}\text { Life Saving } \\
\text { Control }\end{array}$ & $\begin{array}{l}\text { Room and } \\
\text { Equipment }\end{array}$ & $\begin{array}{l}\text { Dangerous } \\
\text { Substance }\end{array}$ & $\begin{array}{c}\text { Medical } \\
\text { Equipment }\end{array}$ & $\begin{array}{l}\text { Eletrical } \\
\text { Handling }\end{array}$ \\
\hline \multirow{3}{*}{$\begin{array}{l}\text { Results } \\
\text { Expectation } \\
\text { Analysis }\end{array}$} & 1.312 & 1.541 & 1.416 & 1.125 & 1.093 & 1.125 & 1.406 \\
\hline & 1.5 & 1.5 & 1.5 & 1.5 & 1.5 & 1.5 & 1.5 \\
\hline & Good & $\begin{array}{c}\text { Not } \\
\text { Good }\end{array}$ & Good & Good & Good & Good & Good \\
\hline
\end{tabular}

\begin{tabular}{lccc}
\hline & Patient Safety & Cupboard Usage & Medicine Management \\
\hline Results & 1.218 & 1.125 & 1.062 \\
Expectation & 1.5 & 1.5 & 1.5 \\
Analysis & Good & Good & Not good \\
\hline
\end{tabular}

Less motivation have impact to staff performance due to patient safety. Training and development program, establish organization culture can be done to increase staff motivation. 6

The policies around fire prevention, life saving control, room and equipments, chemicals and dangerous object safety, management of medical equipments, electrical handling, patient safety, storage cupboard usage and management, all have good policy and procedure. The guideline that is worse than expected is about variable infection control.

This research used self assessment method where all staff gave their judgement related to factors that influenced patient safety in intensive care unit. Hopefully next research could complete this research with bigger sample and more objective method.

\section{Conclusion}

Variable knowledge, attitude, competencies, situation awareness, fatigue, leadership, communiacation, teamwork, teamwork leadership and decision making in ICUSiloam Hospitals Manadoare well performed by the staff, whereas the motivation variable has plenty of room for improvement.

ICU Siloam Hospitals Manado has applied patient safety principles' well. For further improvements, the management should consider to improve staff motivation by training and development program due to achieve better patient safety.

\section{Acknowledgements}

This research is done independently, with no influence or instruction from Siloam Hospitals Manado.

\section{References}

1. Reader, T., Flin, R., Communication skills and error in the intensive care unit, Current Opinions in Critical Care, vol/no: 13(6), pp. 732-736, 2007.

2. Buckley, T., Short, T., Rowbottom, Y., Oh, T., Critical incident reporting in the intensive care unit, Anaesthesia, vol. 52, pp. 403-409, 1997.

3. Osmon, S., et al., Reporting of medical errors: an intensive care unit experience, Crit Care Med, vol/no: 32(3), pp. 727-733, 2004.

4. Donchin, Y., et al., A look into te nature and causes of human errors in the intensive care unit, Critical Care Medicine, vol/no: 23(2), pp. 294-300, 1995.

5. Ayudyawardani, S., Pengembangan model budaya keselamatan pasien yang sesuai di Rumah Sakit Ibu Anak Tumbuh Kembang Cimanggis tahun 2012, FKM UI, 2012. 
6. Robbins S. P., Judge T. A., Perilaku organisasi, buku 1 (ed. 12), Jakarta, Penerbit Salemba empat, pp. 90-266, 2008.

7. Robbins S. P., Judge T. A., Perilaku organisasi, buku 2 (ed. 12), Jakarta, Penerbit Salemba empat, pp. 253-293, 2008. 